\title{
The single-nucleotide polymorphisms in CHD5 affect the prognosis of patients with hepatocellular carcinoma
}

\author{
Xiao Zhu ${ }^{1,10,11, *}$, Qingming Kong ${ }^{2, *}$, Liwei Xie ${ }^{3, *}$, Zhihong Chen ${ }^{1, *}$, Hongmei Li $^{1}$, Zhu \\ Zhu $^{1}$, Yongmei Huang ${ }^{1,4}$, Feifei Lan ${ }^{5}$, Haiqing Luo ${ }^{1,6}$, Jingting Zhan ${ }^{1,4}$, Hongrong \\ Ding $^{1}$, Jinli Lei ${ }^{1,4}$, Qin Xiao ${ }^{7}$, Weiming Fu ${ }^{8}$, Wenguo Fan ${ }^{9}$, Jinfang Zhang ${ }^{12}$ and Hui \\ Luo $^{1,4}$ \\ ${ }^{1}$ Guangdong Provincial Key Laboratory of Medical Molecular Diagnostics, Dongguan Scientific Research Center, Guangdong \\ Medical University, Dongguan, China \\ ${ }^{2}$ Immunity and Biochemical Research Lab, Zhejiang Academy of Medical Sciences, Hangzhou, China \\ ${ }^{3}$ Guangdong Institute of Microbiology, Guangzhou, China \\ ${ }^{4}$ Institute of Marine Medicine Research, Guangdong Medical University, Zhanjiang, China \\ ${ }^{5}$ Forensic Identification Institute, Guangdong Women and Children Hospital, Guangzhou, China \\ ${ }^{6}$ The Affiliated Hospital Cancer Center, Guangdong Medical University, Zhanjiang, China \\ ${ }^{7}$ Department of Blood Transfusion, Peking University Shenzhen Hospital, Shenzhen, China \\ ${ }^{8}$ School of Pharmaceutical Sciences, Southern Medical University, Guangzhou, Guangdong, China \\ ${ }^{9}$ Guanghua School of Stomatology, Hospital of Stomatology, Sun Yat-sen University, Guangzhou, China \\ ${ }^{10}$ Cancer Center, Medical College of Georgia, Georgia Health Sciences University, Augusta, GA, USA \\ ${ }^{11}$ Institute of Bioinformatics, University of Georgia, Athens, GA, USA \\ ${ }^{12}$ Key Laboratory of Orthopaedics and Traumatology, The First Affiliated Hospital of Guangzhou University of Chinese \\ Medicine, The First Clinical Medical College, Guangzhou University of Chinese Medicine, Guangzhou, China \\ *These authors contributed equally to this work \\ Correspondence to: Hui Luo, email: luohui@gdmu.edu.cn \\ Jinfang Zhang, email: zhangjf06@cuhk.edu.hk
}

Keywords: hepatocellular carcinoma; chromodomain-helicase-DNA-binding protein 5; prognosis; single-nucleotide polymorphisms; linkage disequilibrium

Received: May 27, $2017 \quad$ Accepted: November 19, $2017 \quad$ Published: December 26, 2017

Copyright: Zhu et al. This is an open-access article distributed under the terms of the Creative Commons Attribution License 3.0 (CC BY 3.0), which permits unrestricted use, distribution, and reproduction in any medium, provided the original author and source are credited.

\section{ABSTRACT}

Previous studies showed that the low expressions of chromodomain-helicase-DNAbinding protein 5 (CHD5) were intensively associated with deteriorative biologic and clinical characteristics as well as outcomes in many tumors. The aim of this study is to determine whether CHD5 single nucleotide polymorphisms (SNPs) contribute to the prognosis of hepatocellular carcima (HCC). The SNPs were selected according to their linkage disequilibrium (LD) in the targeted next-generation sequencing (NGS) and then genotyped with TaqMan probers. We revealed a rare haplotype AG in CHD5 (SNPs: rs12564469-rs9434711) was markedly associated with HCC prognosis. The univariate and multivariate regression analyses revealed the patients with worse overall survival time were those with tumor metastasis and haplotype AG, as well as cirrhosis, poor differentiation and IV-TNM stage. Based on the available public databases, we discovered the significant association between haplotype AG and CHD5 mRNA expressions only existed in Chinese. These data proposed that the potentially genetic haplotype might functionally contribute to HCC prognosis and CHD5 mRNA expressions.

\section{INTRODUCTION}

Liver cancer is a primary malignancy of the hepar and caused by chronic liver disease and cirrhosis due to hepatitis $\mathrm{B}$, hepatitis $\mathrm{C}$, aflatoxin, alcohol or non-alcoholic fatty liver disease, et al.. The most common types are hepatocellular carcima (HCC), which makes up $80 \%$ of all cases, and cholangiocarcinoma [1]. 
Chromodomain-helicase-DNA-binding protein 5 (CHD5) gene encodes an enzyme that in humans named CHD5 protein. CHD5 is a member of the chromatin organizing modulator domain superfamily, contains two zinc-binding plant homeodomain (PHD) fingers, chromo motifs, and a helicase domain [2]. It has two N-terminal chromodomains and a less well defined C-terminal DNA binding domain, which is approximately 1000 amino acids larger than sequences from members of subfamily I and II [3].

Previous studies suggest that genetic and epigenetic alterations are both involved in inhibiting cell proliferation, migration and invasion, as well as induce apoptosis in some cancer development, such as renal cell carcinoma [4], neuroblastomas [5]. Reduced CHD5 expression is associated with unfavorable clinical features and outcome of cancer patients [6]. Resent studies also showed that CHD5 acted as a tumor suppressor in $\mathrm{HCC}$ [7]. Identification of alterations could be helpful to unravel the mechanisms underlying carcinogenesis and develop potential biomarkers for cancer screening and prognosis prediction. This work will study the association of its single-nucleotide polymorphisms (SNPs) and HCC patients' prognosis was not studied yet.

\section{RESULTS}

\section{Univariate and multivariate regression models of prognostic factors}

Five-year overall survivals for patients were $7.50 \%$ in the discovery study, $8.38 \%$ in the replication study, and $8.08 \%$ in the combined study. We investigated the association of patients' overall survival. Patients with haplotype AG had a shorter survival time (median 17.00 months) in the discovery, replication and combined studies, with conspicuous log-rank $P$ values $\left(3.673 \times 10^{-}\right.$ ${ }^{7}$, cases in the discovery study; $5.000 \times 10^{-6}$, cases in the replication study; and $1.437 \times 10^{-11}$, cases in the combined study) (Figure 1). We analyzed the associations of overall survival and the clinical variables, and found age, cirrhosis, differentiation, metastasis and TNM stages were associated with HCC survival in univariate analysis (Table 1).

For further validating the importance of the regression variables, a re-estimation of multivariate model analysis was carried out for the above 8 characteristics in the discovery, replication and/or combined studies. We found only the percentage of age made a reverse significant but marginal contribution $(\geq 55 v s .<55$ : $\mathrm{OR}=0.87<$ $1, P=0.047)$. We found tumor metastasis, haplotype AG, cirrhosis, poor differentiation and IV-TNM stage were the prognostic factors of $\mathrm{HCC}(\mathrm{OR}>1, P<0.05$; Table 2$)$.

\section{Evolutional conserved regions (ECRs), mutations, expressions of CHD5 in silico}

On the basis of the information in UCSC database and NCBI, the Homo sapiens CHD5 gene is located on chromosome 1p36.31 (Figure 2A), and its transcript is composed of 42 exons (which encodes a protein of 1954 amino acids) (Figure 2B). We identified an ECR by local and global alignment programs, which indicated that $C H D 5$ is evolutionarily conserved (especially in the exons) among diverse species (Figure 2C).

When looking at the incidence of mutations in the CHD5 encoding the protein in tumor samples, using TCGA and the web tool cBioPortal for visualization and analysis, we identified a total of 11 mutations in CHD5 mRNA from TCGA dataset, consisting of 8 missense and 3 truncating mutations. Eight tumor samples (H112501, H072969, TCGA-BC-A10W-01, TCGA-G3-AAV0-01, TCGA-DD-AADF-01, TCGA-DD-AACQ-01, TCGAGJ-A9DB-01 and TCGA-G3-A25Z-01) had a different missense mutation, one sample (TCGA-4R-AA8I-01) had a splice mutation (X812_splice), one sample (TCGA-DDA1EG-01) had a deletion (D783Tfs*7), and one sample (TCGA-CC-A7IK-01) had a nonsense mutation (W591*). Of them, three of the mutations in the CHD5 gene are localized to the amino-median of the SNF2_N domain (Figure 2B). And the deletions were only found in the data from the National Cancer Institute in HCC samples, which did not found in the East Asian patients (Figure 2D). In Figure 2E, copy-number alterations from GISTIC [8] showed those who have shallow deletion and diploid have higher CHD5 expressions in the Western population. Compared with the normal samples (paracarcinoma tissues), HCC tissues had a higher expression $(P<0.005$; Figure 2F).

\section{Correlating block 3 haplotypes and phenotype to predict HCC prognosis}

According to the next generation RNA-seq from TCGA, we found that CHD5 expressed in almost all human normal tissues, cells and fluids (Supplementary Figure 1). In the haplotype-phenotype correlation analysis, we found statistically significant trends for the haplotypes effect on CHD5 mRNA expression in CHB (45 unrelated Han Chinese in Beijing, $P_{\mathrm{AG} / \mathrm{AA}}=0.041, P_{\mathrm{AG} / \mathrm{GG}}=0.027, P_{\text {trend }}$ $=0.017)$. But the same trend was not found in JPT (45 unrelated Japanese in Tokyo, $\left.P_{\text {trend }}=0.194\right)$, CEU (90 Utah residents from northern and western Europe, $P_{\text {trend }}=0.425$ ), and YRI (90 Yoruba in Ibadan, Nigeria, $P_{\text {trend }}^{\text {trend }}=0.737$; Figure 3). This also suggested that CHD5 block 3 might be a causative loci and therapeutic target for HCC in Chinese.

\section{DISCUSSION}

The progression of HCC from diagnosis to death is double-quick, with patients dying within several months after diagnosis. This disease is almost asymptomatic in the early stages and the patients are unaware of the disease until the later stages, which the tumor has already metastasized. Most of the patients cannot undergo surgery 
Table 1: Clinical and laboratory features of the subjects and univariate analysis for overall survival

\begin{tabular}{|c|c|c|c|c|c|c|c|c|c|}
\hline \multirow[t]{2}{*}{ Variables } & \multicolumn{3}{|c|}{ Discovery study } & \multicolumn{3}{|c|}{ Replication study } & \multicolumn{3}{|c|}{ Combined study } \\
\hline & $n$ & $\begin{array}{l}5 \text {-years } \\
\text { survival } \\
\text { rates \% }\end{array}$ & $\begin{array}{l}\text { Log- } \\
\text { rank } P\end{array}$ & $n$ & $\begin{array}{l}5 \text {-years } \\
\text { survival } \\
\text { rates \% }\end{array}$ & $\begin{array}{l}\text { Log-rank } \\
P\end{array}$ & $n$ & $\begin{array}{l}5 \text {-years } \\
\text { survival } \\
\text { rates \% }\end{array}$ & Log-rank $P$ \\
\hline \multicolumn{10}{|l|}{ Age (years) } \\
\hline$<55$ & 98 & 5.10 & & 213 & 4.23 & & 311 & 4.50 & \\
\hline$\geq 55$ & 182 & 8.79 & 0.087 & 336 & 11.01 & 0.036 & 518 & 10.23 & 0.013 \\
\hline \multicolumn{10}{|l|}{ Gender } \\
\hline Females & 53 & 9.43 & & 125 & 9.60 & & 178 & 9.55 & \\
\hline Males & 227 & 7.05 & 0.405 & 424 & 8.02 & 0.747 & 651 & 7.68 & 0.584 \\
\hline \multicolumn{10}{|l|}{ Smoking } \\
\hline No & 176 & 7.95 & & 296 & 10.47 & & 472 & 9.53 & \\
\hline yes & 99 & 7.07 & 0.942 & 231 & 6.49 & 0.183 & 330 & 6.67 & 0.297 \\
\hline \multicolumn{10}{|l|}{ Drinking } \\
\hline No & 177 & 8.47 & & 311 & 11.25 & & 488 & 10.25 & \\
\hline Yes & 95 & 6.32 & 0.401 & 210 & 5.24 & 0.059 & 305 & 5.57 & 0.131 \\
\hline \multicolumn{10}{|l|}{$\mathrm{HBV}$} \\
\hline HBsAg (-) & 56 & 10.71 & & 130 & 12.30 & & 186 & 11.83 & \\
\hline HBsAg $(+)$ & 224 & 6.70 & 0.141 & 419 & 7.16 & 0.131 & 643 & 7.00 & 0.136 \\
\hline \multicolumn{10}{|l|}{ Serum AFP } \\
\hline$<25 \mathrm{ng} / \mathrm{ml}$ & 47 & 6.38 & & 118 & 10.17 & & 165 & 9.09 & \\
\hline$\geq 25 \mathrm{ng} / \mathrm{ml}$ & 233 & 7.73 & 0.869 & 431 & 7.89 & 0.495 & 664 & 7.83 & 0.623 \\
\hline \multicolumn{10}{|l|}{ Tumor size $(\mathrm{cm})$} \\
\hline$\leq 5$ & 65 & 10.77 & & 139 & 11.51 & & 204 & 11.27 & \\
\hline$>5, \leq 10$ & 93 & 8.60 & 0.538 & 273 & 7.33 & 0.169 & 366 & 7.65 & 0.215 \\
\hline$>10$ & 122 & 4.92 & 0.037 & 137 & 7.30 & 0.104 & 259 & 6.18 & 0.058 \\
\hline \multicolumn{10}{|l|}{ Cirrhosis } \\
\hline No & 16 & 25.00 & & 38 & 15.79 & & 54 & 18.52 & \\
\hline Yes & 260 & 6.54 & 0.005 & 504 & 7.94 & 0.027 & 764 & 7.46 & $<0.001$ \\
\hline \multicolumn{10}{|l|}{ Tumor morphology } \\
\hline No residual tumor & 19 & 15.79 & & 43 & 13.95 & & 62 & 14.52 & \\
\hline Uninodular tumor & 55 & 9.09 & 0.192 & 89 & 8.99 & 0.744 & 144 & 9.03 & 0.376 \\
\hline $\begin{array}{l}\text { Multinodular } \\
\text { tumor }\end{array}$ & 107 & 7.48 & 0.111 & 228 & 7.02 & 0.078 & 335 & 7.16 & 0.090 \\
\hline Massive tumor & 92 & 5.43 & 0.035 & 168 & 9.52 & 0.301 & 260 & 8.08 & 0.124 \\
\hline \multicolumn{10}{|l|}{ Differentiation } \\
\hline Well & 31 & 48.39 & & 77 & 25.97 & & 108 & 32.41 & \\
\hline Moderate & 78 & 3.85 & $<0.001$ & 195 & 7.69 & $<0.001$ & 273 & 6.59 & $<0.001$ \\
\hline Poor & 171 & 1.75 & $<0.001$ & 277 & 3.97 & $<0.001$ & 448 & 3.13 & $<0.001$ \\
\hline \multicolumn{10}{|l|}{ Metastasis } \\
\hline Abscent & 81 & 22.22 & & 189 & 19.58 & & 270 & 20.37 & \\
\hline Present & 193 & 1.55 & $<0.001$ & 347 & 2.59 & $<0.001$ & 540 & 2.22 & $<0.001$ \\
\hline \multicolumn{10}{|l|}{ TNM stage } \\
\hline I & 53 & 16.98 & & 148 & 14.86 & & 201 & 15.42 & \\
\hline II & 95 & 7.37 & 0.039 & 230 & 6.52 & 0.051 & 325 & 6.77 & 0.025 \\
\hline III & 64 & 4.69 & 0.006 & 110 & 5.45 & 0.025 & 174 & 5.17 & 0.004 \\
\hline IV & 68 & 2.94 & $<0.001$ & 61 & 4.92 & 0.001 & 129 & 3.88 & $<0.001$ \\
\hline
\end{tabular}

AFP, alpha fetoprotein; TNM, tumor, node, metastasis-classification.

because the tumors are too advanced for resection when diagnosed. Also, the recurrence rate of this tumor is very high after surgery [9].

With the recent advances in molecular biology, important discoveries were found. As a chromatin- remodeling protein, CHD5 could bind DNA through histones and regulate gene transcription. It could positively regulate $\mathrm{H} 3 \mathrm{~K} 27 \mathrm{me} 3$ thereby precisely inhibiting genes that promote the cell proliferation and differentiation in nerve cells or non-neuronal cells [10]. Furthermore, the 
Table 2: Cox multivariate regression of potential prognostic factors for overall survival

\begin{tabular}{|c|c|c|c|c|c|c|}
\hline \multirow[t]{2}{*}{ Variables } & \multicolumn{2}{|l|}{ Discovery study } & \multicolumn{2}{|l|}{ Replication study } & \multicolumn{2}{|l|}{ Combined study } \\
\hline & HR $(95 \%$ CI $)$ & $P$ & HR $(95 \% C I)$ & $\boldsymbol{P}$ & HR $(95 \% C I)$ & $P$ \\
\hline Age $($ years $) \geq 55$ vs. $<55$ & l & I & $0.95(0.86-1.07)$ & 0.197 & $0.87(0.77-0.98)$ & 0.047 \\
\hline Tumor size $>10 \mathrm{~cm}$ vs. $\leq 5 \mathrm{~cm}$ & $1.02(0.83-1.23)$ & 0.563 & 1 & 1 & 1 & 1 \\
\hline Cirrhosis Yes vs. No & $1.32(1.16-1.49)$ & 0.016 & $1.10(1.01-1.28)$ & 0.049 & $1.82(1.19-2.72)$ & 0.008 \\
\hline \multicolumn{7}{|l|}{ Tumor morphology } \\
\hline Massive tumor vs. No residual tumor & $1.06(0.86-1.28)$ & 0.404 & / & l & l & / \\
\hline \multicolumn{7}{|l|}{ Differentiation } \\
\hline Moderate vs. Well & $1.03(0.75-1.44)$ & 0.585 & $1.74(0.96-1.25)$ & 0.133 & $1.34(0.83-1.60)$ & 0.127 \\
\hline Poor vs. Moderate & $6.09(3.71-8.85)$ & $8.062 \times 10^{-4}$ & $2.57(1.37-3.92)$ & 0.009 & $5.94(2.11-9.52)$ & 0.002 \\
\hline Metastasis Present vs. Abscent & $8.52(3.19-16.37)$ & $1.990 \times 10^{-4}$ & $5.76(2.54-10.71)$ & $9.175 \times 10^{-4}$ & $14.08(2.88-31.38)$ & $2.636 \times 10^{-5}$ \\
\hline \multicolumn{7}{|l|}{ TNM stage } \\
\hline II vs. I & $1.09(0.90-1.34)$ & 0.179 & 1 & I & $1.15(0.96-1.28)$ & 0.064 \\
\hline III vs. I & $1.17(0.97-1.41)$ & 0.074 & $1.12(0.92-1.29)$ & 0.124 & $1.45(0.95-1.80)$ & 0.093 \\
\hline IV vs. I & $3.35(1.23-5.69)$ & 0.006 & $2.95(1.25-4.73)$ & 0.017 & $3.72(1.45-5.95)$ & 0.009 \\
\hline \multicolumn{7}{|l|}{ Haplotype (block 3) } \\
\hline AG vs. $\mathrm{AA}+\mathrm{GG}$ & $6.58(3.56-9.55)$ & $7.827 \times 10^{-4}$ & $5.21(2.34-8.17)$ & 0.003 & $11.86(3.37-21.14)$ & $8.569 \times 10^{-5}$ \\
\hline
\end{tabular}

downstream genes activated may include CDKN2A that positively regulates the p53/TP53 pathway, which in turn, prevents cell proliferation $[11,12]$.

Previous studies revealed that truncation mutations of CHD5 occur in neuroblastomas [13]. For this reason, we predicted that mutations in the PHD might lead to the loss or gain of function of CHD5 proteins, thereby influencing patients' overall survival and prognosis. Therefore, we studied the overall survival in two cases and combined them.

We found a significant trend for the effect of haplotype AG on CHD5 transcript expressions in Han Chinese but not other populations, indicating that this haplotype may be a potential genetic determinant and therapeutic target for $\mathrm{HCC}$ in Chinese. Haplotype AG (block 3) was strikingly associated with a poor prognosis in HCC patients. Forward progressive selection-univariate analysis, using the training set, resulted in the options of the coefficients, all with $P$ values $<0.05$. In the present study, we found that the prognosis of patients metastatic diseases with haplotype AG is no better than that of patients without metastasis and with other haplotypes. Haplotypes are a set of alleles of a group of closed linked genetic markers, which are usually inherited as a unit [14]. An individual inherited a complete haplotype from each parent, therefore could narrow down the numbers of investigated markers.

Further genotype-phenotype analyses showed that the lower level of CHD5 mRNA expression was associated with the haplotype $\mathrm{AG}$ in Chinese population but not in Japanese, Caucasian and African populations. Given that lower CHD5 mRNA expression levels were correlated to haplotype AG in some target tissues, joined the above survival analyses, it is reasonable to surmise that lower
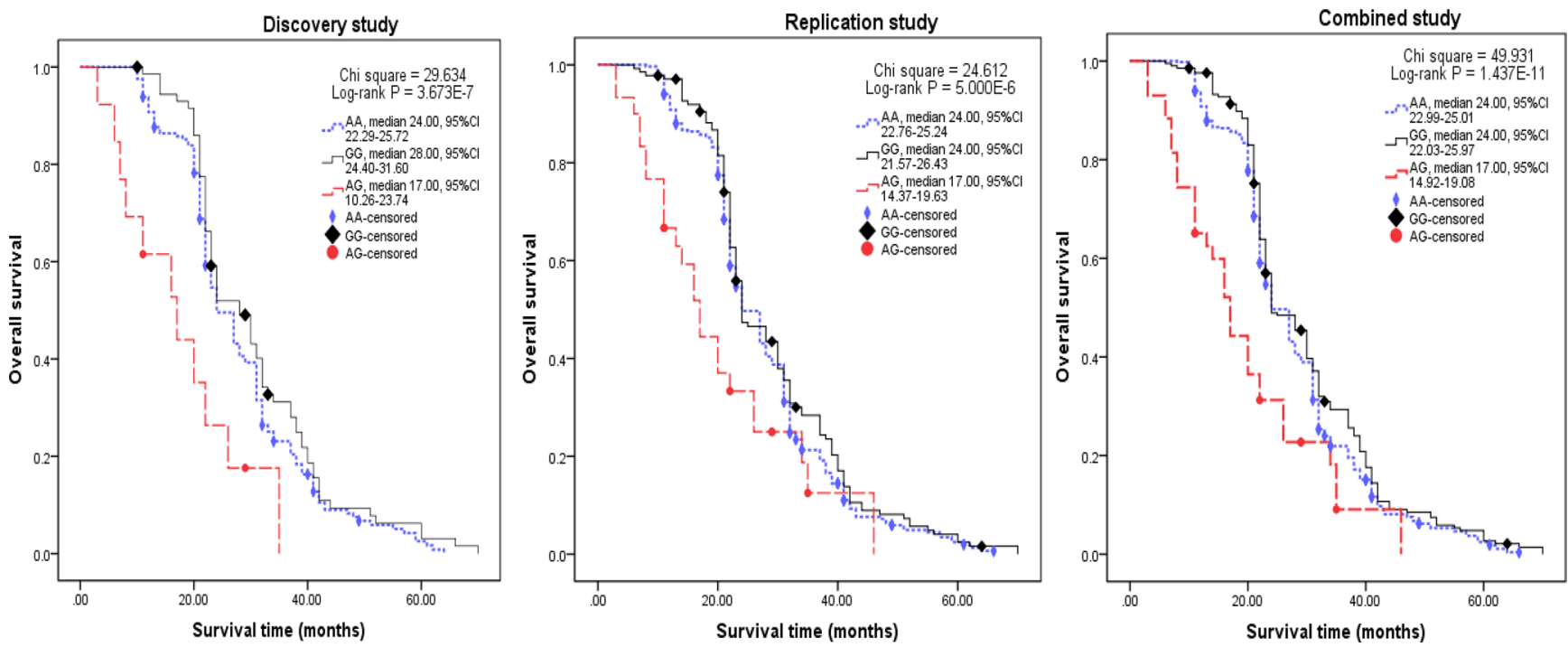

Figure 1: Kaplan-Meier survival curves according to haplotypes (block 3 ) in the discovery, replication and combined studies. $P$ value was calculated using a log-rank test. 
CHD5 mRNA expression levels may be associated with shorter survival time in Chinese populations. However, the underlying mechanisms are still unintelligible. Recent studies have showed that CHD5 expression is silenced by epigenetical hypermethylation in the gene promoter in some tumors including HCC and gastric cancer $[7,15,16]$.
The CHD5 hypermethylation may implicate chromatin dynamics and cancer-associated pathways. The hypothesis that CHD5 works as a tumor-suppressing gene in HCC might simplify the tanglesome landscape of a chromatin remodeler. Many of these enzymes, such as CHD4 and BRG1, including their mutations and SNPs, display

A

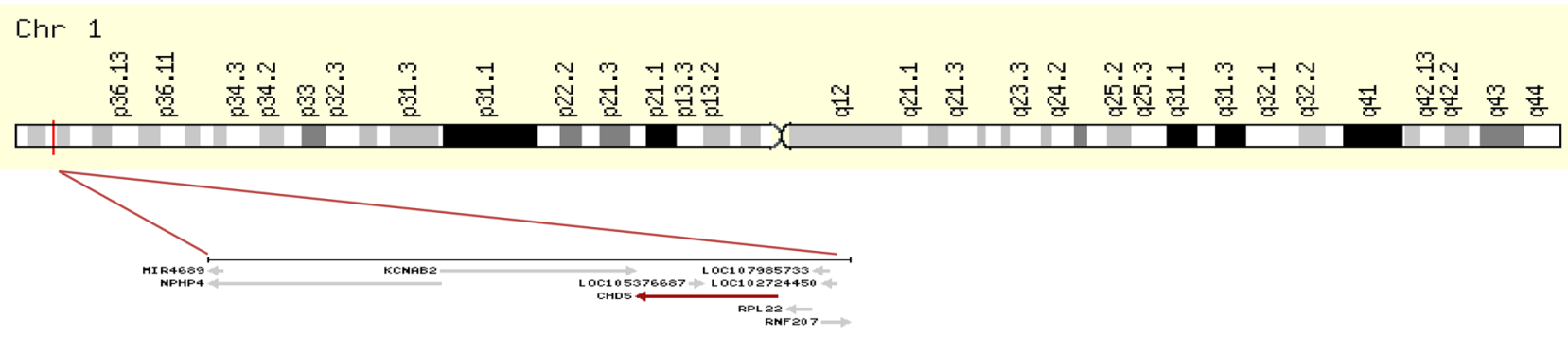

B

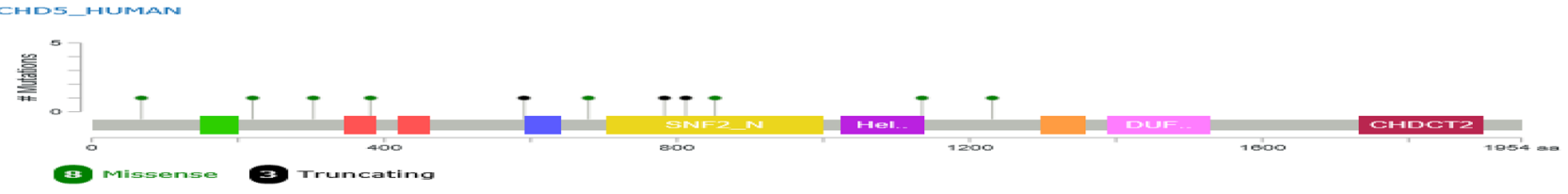

C

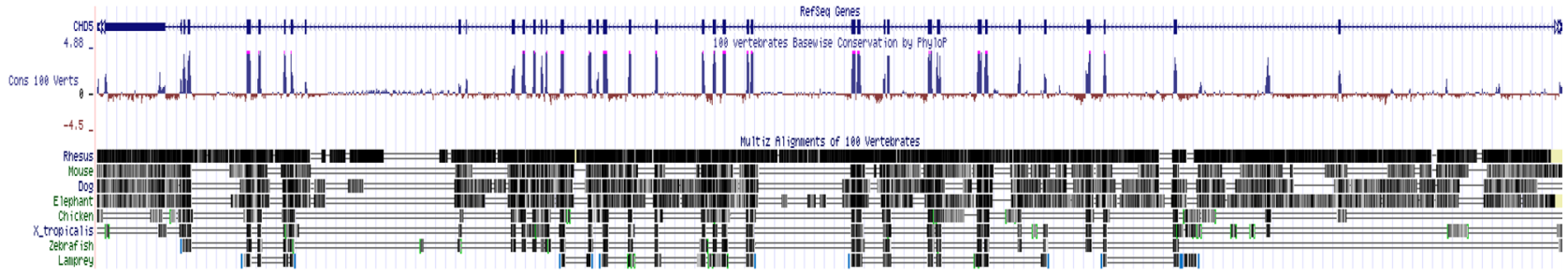

D

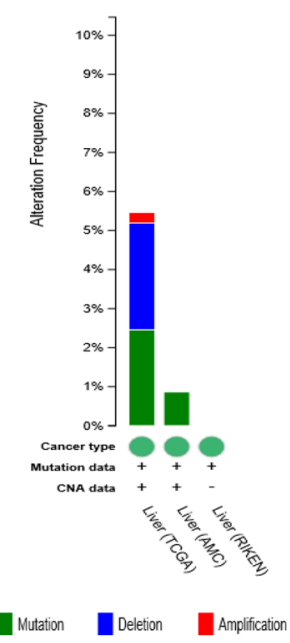

$\mathbf{E}$

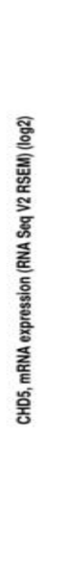

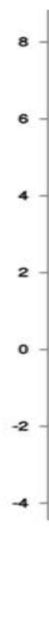

-

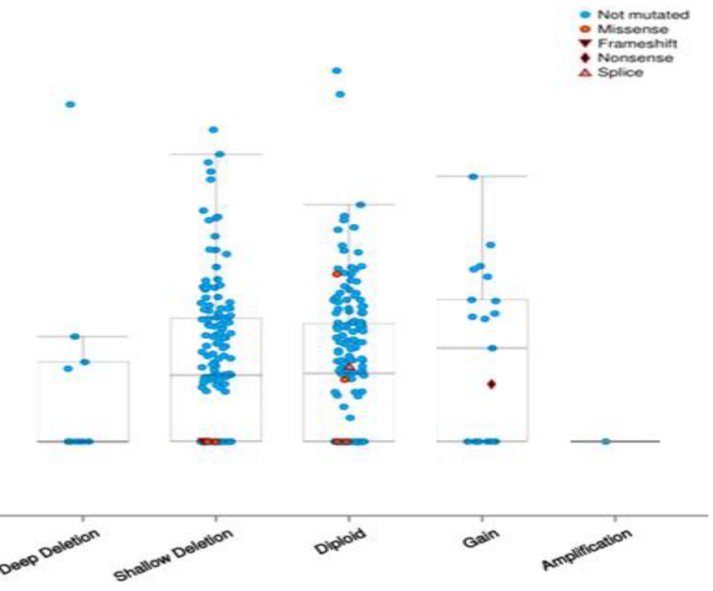

CHD5, Putative copy-number alterations trom GISTIC

F

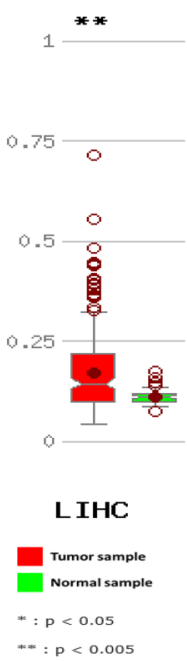

Figure 2: CHD5 structure, mutations and expressions in silico. (A) CHD5 in chromosome 1p36.31 and its transcriptional direction from the National Center for Biotechnology Information (NCBI, https://www.ncbi.nlm.nih.gov/). (B) CHD5 gene mutations from TCGA (https://cancergenome.nih.gov/). The Cancer Genome Atlas (TCGA) hepatocellular carcinoma datasets and the related clinicopathologic information of the included patients were obtained from the cBioPortal (http://www.cbioportal.org/) for Cancer Genomics generated by Memorial Sloan-Kettering Cancer Center. (C) CHD5 gene ECR generated from the UCSC Genome Browser (https://genome.ucsc.edu/). (D) the deletions of CHD5 from TCGA in silico analysis. (E) copy-number alterations from GISTIC (https://software.broadinstitute. org/software/cprg/?q = node/31). By separating somatic copy-number alterations (SCNAs) profiles into underlying arm-level and focal alterations, GISTIC estimates the background rates for each category as well as defines the boundaries of SCNA regions. (F) CHD5 expressions in HCC samples and matched normal samples comparing of average beta value from TCGA. 
ambiguous roles during oncogenesis. As a chromatin remodeling protein, CHD5 could regulate developmentally ATP-dependent chromatin remodeling [17]. The function change of protein may influence on patients survival time.

We do not yet conclude the mechanisms of how it is impacting on the expression of CHD5 protein in HCC tissues. Taken these limitations into consideration, we recognized that further investigations in HCC patients needed to be implemented to find out the precise mechanisms.

\section{MATERIALS AND METHODS}

\section{Ethics statement}

The study was approved by the local ethics committee (Guangdong Medical University). All the relatives of patients provided written informed consent. The study was conducted according to the principles expressed in the Declaration of Helsinki.
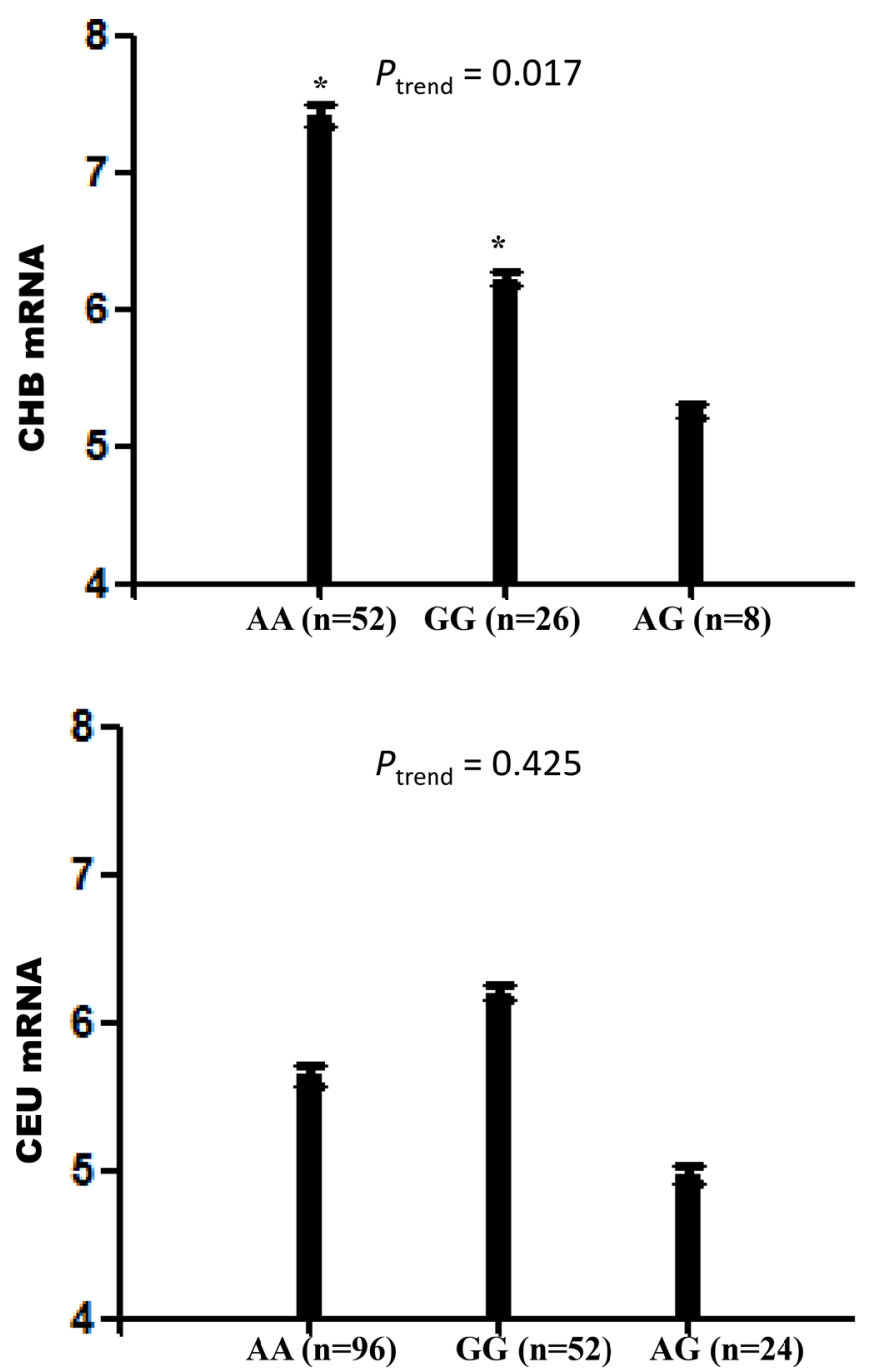

\section{Study subjects}

Firstly, 280 unrelated HCC patients (who were from Zibo Central Hospital in North China between 2006 and 2010) were recruited in the discovery study. Then, 549 HCC patients (who were from Peking University Shenzhen Hospital between 2007 and 2010, the First Affiliated Hospital, Sun Yat-Sen University between 2007 and 2015, and Cancer Hospital of Guangzhou Medical University between 2009 and 2011 in South China) were included in the replication study. The mean age (years) of the patients was all around 56 in two groups. Age distribution was throughout 30 to 75 years old. Furthermore, the ratio of male individuals is about 3.65 times higher than that of females in cases (combined study). All cases were Han origin Chinese and lived in China. The main features of the subjects included are summarized in Table 1.

At recruitment, each study participant (or his/her relative) was interviewed via a structured questionnaire, to obtain information on demographic characteristics,
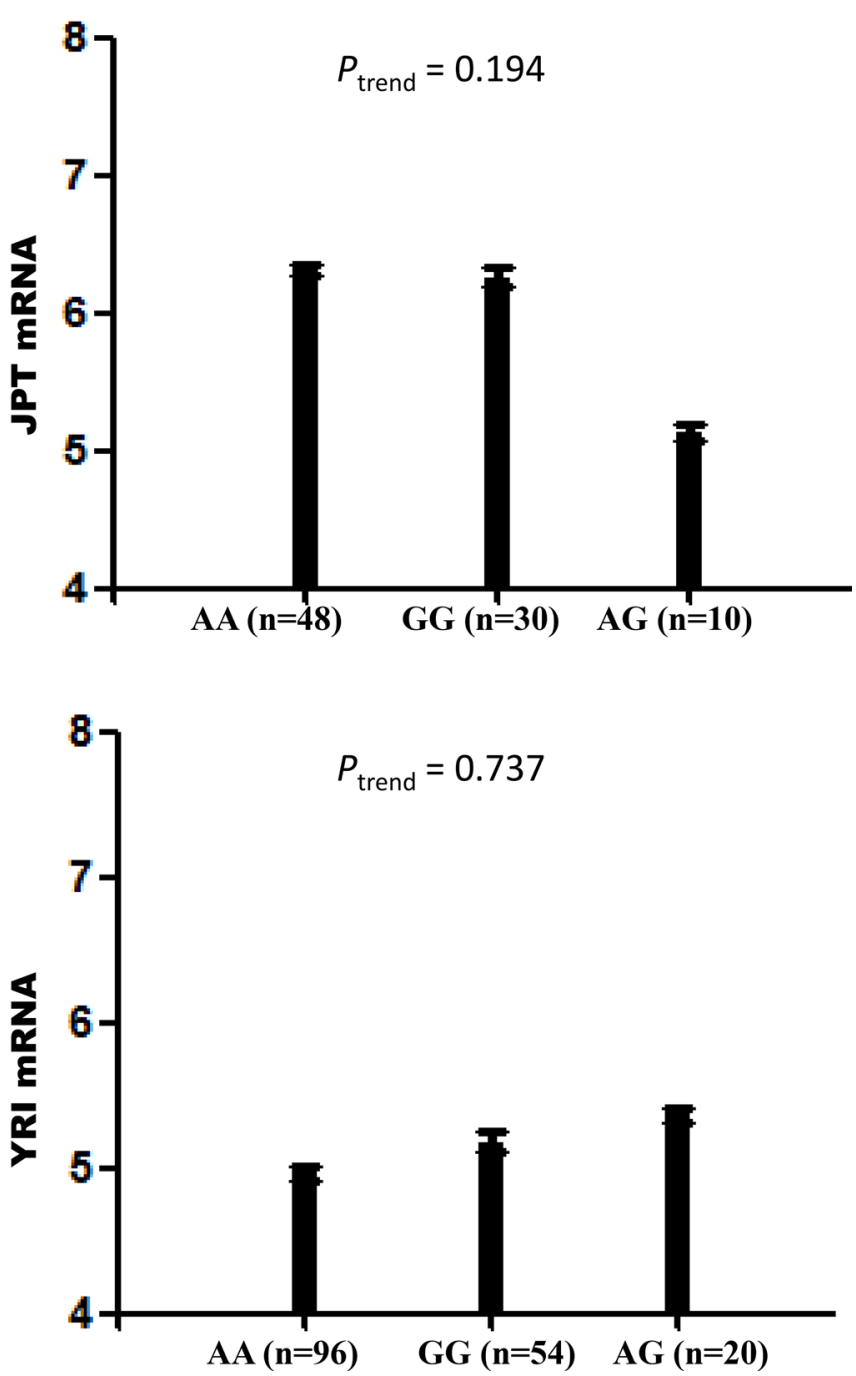

Figure 3: Correlation between CHD5 mRNA expressions and haplotypes (block 3) from EBV-transformed B lymphoblastoid cell lines from different populations available by SNPexp. 
habits of alcohol drinking and cigarette smoking, as well as personal and family history of major chronic illnesses. Pack-years were calculated as the average number of pack of cigarettes consumed per day multiplied by the number of years a person has smoked [18]. We defined "former smokers" as the above who quitted smoking $\geq$ 1 year previously, and "current smokers" as the above who currently smoked or quitted smoking $<1$ year ahead of the interview. We defined "never smokers" as those who had never smoked or had smoked for $<1$ year. Ever drinkers who had stopped drinking more than one year previously were regarded as "former drinkers" and the others as "current drinkers". The amount of each type of beverage (liquor/spirits, wine or beer) consumed during the year before the registration was reported. Daily ethanol consumption in grams was calculated rooted in the ethanol content of the beverage. One drink was regarded as $30 \mathrm{~g}$ of spirits (12.9 $\mathrm{g}$ of ethanol), $103 \mathrm{~g}$ of wine (12.3 $\mathrm{g}$ of ethanol), or $360 \mathrm{~g}$ of beer (12.6 $\mathrm{g}$ of ethanol) $[19,20]$. The number of subjects in cases who drunk and/or used alcohol was much more than that in controls. Several studies on the tobacco or alcohol consuming in HCC patients have been conducted with conflicting results. In our research, the proportion of smoking or drinking has about $39.81 \%$ or $36.79 \%$ in HCC patients (combined study). However, if we want to know the effects of smoking or drinking in HCC, further studies in larger samples should be managed.

The serum laboratory tests, tumor characteristics, staging and prior therapy were collected according to the records of case history. The diagnostic criteria of HCC have been described as our previous study [21]. Briefly, the diagnosis of HCC was verified by either positive liver histologic findings, or rooted in the findings of medical imaging features indicative of $\mathrm{HCC}$ in at least two image examinations including abdominal ultrosound, highresolution contrast enhanced computed tomography (CT), magnetic resonance imaging (MRI) and liver angiography, or by a single positive imaging technique added to serum $\alpha$-fetoprotein level $\geq 25 \mu \mathrm{g} / \mathrm{L}$. Those negative results in abdominal iconography or $\alpha$-fetoprotein levels will be diagnostic by transparietal biopsy proof. The tumor, nodes, metastasis-classification (TNM) system was described as before [22].

\section{Patients follow up}

Survival Data were gathered from the inpatient and outpatient records in the hospitals, primary physician's offices, and/or patient or family contact. The duration of survival was from the date of carcinomatous diagnosis to the date of death, or last known date alive. The patients were followed up for a median time of five years. The log-rank test was used to judge the relationship between the haplotypes (block 3) and the prognosis of patients from the date of diagnosis to the end of follow-up. The
Cox regression model was used to analyzed survival-time (time-to-event) outcomes on one or more predictors.

\section{Targeted sequencing, SNPs selection and genotyping}

We sequenced whole CHD5 gene with next generation sequencing technology (Illumina Genome Analyzer) in $280 \mathrm{HCC}$ samples and found total 164 SNPs in this gene including its 5'- and 3'-ends (Supplementary Figure 2). We defined haplotype blocks according to linkage disequilibrium (LD) using the Haploview program [23]. The SNPs selected following the haplotype blocks were rs12037962, rs11587, rs41307753 and rs3810989 (in block 1), rs2273041, rs2273040, rs2273038 and rs55930553 (in block 2), and rs12564469 and rs9434711 (in block 3) (Supplementary Figure 2). Then, genomic DNAs from all the other subjects (549 cases) were genotyped by TaqMan probes in Applied Biosystems ABI 7500 Fast System (Forster City, CA) for the selected two SNPs in haplotypic block 3 (rs12564469 and rs9434711).

\section{Comparative genomic analysis of CHD5 gene}

To identify evolutionarily conserved regions (ECRs), we compared the Homo sapiens CHD5 sequence (UCSC Genome Browser on Human Dec. 2013 (GRCh38/ hg38) Assembly) with 44 vertebrate homologous sequences. The BLASTZ algorithm [24] and PhastCons [25] incorporated in the UCSC browser [26].

\section{Data mining of CHD5 mutations/deletions and mRNA expressions in HCCs}

To portray the regulation model of CHD5 gene, the CHD5 mRNA expression data from The Cancer Genome Atlas (TCGA) hepatocellular carcinoma datasets and the related clinicopathologic information of the included patients were obtained from the cBioPortal for Cancer Genomics generated by Memorial Sloan-Kettering Cancer Center. For mRNA expression data, the relative expression of an individual gene and the distribution of a gene's expression in a reference population were analyzed. Analysis of 442 HCC samples (TCGA, provisional), 231 HCC samples (from Asian Medical Center, Korea) [27], and $27 \mathrm{HCC}$ samples (from RIKEN with whole-genome sequencing) [28] were performed in silico.

\section{Haplotype-phenotype correlation analysis}

CHD5 expressions data in normal human tissues, cells and fluids were analyzed from the next-generation sequencing (GREx/Illumina Human BodyMap from TCGA). To further understand how the CHD5 genetic variation influences its expression in tumor progress, we tried to examine the data from the HapMap Project consisting of 3.96 million SNP genotypes from 270 
individuals of four ethnic groups (CHB, JPT, CEU, and YRI) and CHD5 mRNA expression levels from EBVtransformed B lymphoblastoid cell lines from the same 270 individuals available by SNPexp [29]. Finally, least squares analysis of variance (ANOVA) was performed to calculate statistical levels of CHD5 transcripts expression according to different haplotypes.

\section{Statistical analysis}

All statistical tests were two-sided and $P$ values less than 0.05 were considered statistically significant. SPSS 22.0 (SPSS, Chicago, IL) was used in this study. Kaplan-Meier survival curves and the log-rank test for trend were used to evaluate the relationship between the potential haplotypes and the HCC prognosis. Hazard ratios (HR) and 95\% confidence intervals (CIs) for each factor in multivariate analysis were calculated from the Cox regression model.

\section{ACKNOWLEDGMENTS AND FUNDING}

This work was supported by National Natural Science Foundation of China (81541153); Guangdong Provincial Science and Technology Programs (2015A050502048, 2016A050503046, 2014A020212295, and 2014A020212653); Science and Technology Research Project in Dongguan City (2013508152011 and 2013508152002). We greatly acknowledge the study participants and their families. We are grateful for the collaboration received from the participating hospitals and the staff.

\section{CONFLICTS OF INTEREST}

The authors have no conflicts of interest to declare.

\section{REFERENCES}

1. Siegel RL, Miller KD, Jemal A. Cancer statistics, 2016. CA Cancer J Clin. 2016; 66:7-30.

2. Mallette FA, Richard S. JMJD2A Promotes Cellular Transformation by Blocking Cellular Senescence through Transcriptional Repression of the Tumor Suppressor CHD5. Cell reports. 2012; 2:1233-1243.

3. Schuster EF, Stoger R. CHD5 defines a new subfamily of chromodomain-SWI2/SNF2-like helicases. Mamm Genome. 2002; 13:117-119.

4. Du Z, Li L, Huang X, Jin J, Huang S, Zhang Q, Tao Q. The epigenetic modifier CHD5 functions as a novel tumor suppressor for renal cell carcinoma and is predominantly inactivated by promoter CpG methylation. Oncotarget. 2016; 7:21618-21630. https://doi.org/10.18632/oncotarget.7822.

5. Naraparaju K, Kolla V, Zhuang T, Higashi M, Iyer R, Kolla S, Okawa ER, Blobel GA, Brodeur GM. Role of
microRNAs in epigenetic silencing of the CHD5 tumor suppressor gene in neuroblastomas. Oncotarget. 2016; 7:15977-15985. https://doi.org/10.18632/oncotarget.7434.

6. Hall WA, Petrova AV, Colbert LE, Hardy CW, Fisher SB, Saka B, Shelton JW, Warren MD, Pantazides BG, Gandhi K, Kowalski J, Kooby DA, El-Rayes BF, et al. Low CHD5 expression activates the DNA damage response and predicts poor outcome in patients undergoing adjuvant therapy for resected pancreatic cancer. Oncogene. 2014; 33:5450-5456.

7. Zhao R, Wang N, Huang H, Ma W, Yan Q. CHD5 a tumour suppressor is epigenetically silenced in hepatocellular carcinoma. Liver Int. 2014; 34:e151-160.

8. Mermel CH, Schumacher SE, Hill B, Meyerson ML, Beroukhim R, Getz G. GISTIC2.0 facilitates sensitive and confident localization of the targets of focal somatic copynumber alteration in human cancers. Genome Biol. 2011; 12:R41.

9. Kim G, Jang SY, Han E, Lee YH, Park SY, Nam CM, Kang ES. Effect of statin on hepatocellular carcinoma in patients with type 2 diabetes: A nationwide nested case-control study. Int J Cancer. 2017; 140:798-806.

10. Xie CR, Li Z, Sun HG, Wang FQ, Sun Y, Zhao WX, Zhang S, Wang XM, Yin ZY. Mutual regulation between CHD5 and EZH2 in hepatocellular carcinoma. Oncotarget. 2015; 6:40940-40952. https://doi.org/10.18632/oncotarget.5724.

11. Ng D, Yang XR, Tucker MA, Goldstein AM. Mutation screening of CHD5 in melanoma-prone families linked to 1 p36 revealed no deleterious coding or splice site changes. BMC Res Notes. 2008; 1:86.

12. Cai C, Ashktorab H, Pang X, Zhao Y, Sha W, Liu Y, Gu X. MicroRNA-211 expression promotes colorectal cancer cell growth in vitro and in vivo by targeting tumor suppressor CHD5. PLoS One. 2012; 7:e29750.

13. Fujita T, Igarashi J, Okawa ER, Gotoh T, Manne J, Kolla V, Kim J, Zhao H, Pawel BR, London WB, Maris JM, White PS, Brodeur GM. CHD5, a tumor suppressor gene deleted from 1p36.31 in neuroblastomas. J Natl Cancer Inst. 2008; 100:940-949.

14. Gabriel SB, Schaffner SF, Nguyen H, Moore JM, Roy J, Blumenstiel B, Higgins J, DeFelice M, Lochner A, Faggart M, Liu-Cordero SN, Rotimi C, Adeyemo A, et al. The structure of haplotype blocks in the human genome. Science. 2002; 296:2225-2229.

15. Kolla V, Zhuang T, Higashi M, Naraparaju K, Brodeur GM. Role of CHD5 in human cancers: 10 years later. Cancer Res. 2014; 74:652-658.

16. Wang X, Lau KK, So LK, Lam YW. CHD5 is downregulated through promoter hypermethylation in gastric cancer. J Biomed Sci. 2009; 16:95.

17. Potts RC, Zhang P, Wurster AL, Precht P, Mughal MR, Wood WH $3^{\text {rd }}$, Zhang Y, Becker KG, Mattson MP, Pazin MJ. CHD5, a brain-specific paralog of Mi2 chromatin remodeling enzymes, regulates expression of neuronal genes. PLoS One. 2011; 6:e24515. 
18. Garces YI, Yang P, Parkinson J, Zhao X, Wampfler JA, Ebbert JO, Sloan JA. The relationship between cigarette smoking and quality of life after lung cancer diagnosis. Chest. 2004; 126:1733-1741.

19. Adames C. Nutritive value of American foods in common unit. Agriculture Handbook No 456 Washington, DC: US Department of Agriculture, US Government Printing Office. 1975; 31.

20. Yuan JM, Ross RK, Gao YT, Henderson BE, Yu MC. Follow up study of moderate alcohol intake and mortality among middle aged men in Shanghai, China. BMJ. 1997; 314:18-23.

21. Zhu X, Zhang J, Fan W, Wang F, Yao H, Wang Z, Hou $\mathrm{S}$, Tian Y, Fu W, Xie D, Zhu W, Long J, Wu L, et al. The rs391957 variant cis-regulating oncogene GRP78 expression contributes to the risk of hepatocellular carcinoma. Carcinogenesis. 2013; 34:1273-1280.

22. Valls C, Figueras J, Pamies JJ, Virgili J, Sancho C, Dominguez J, Montana X, Benasco C, Casais L, Jaurrieta E. Preoperative TNM staging of hepatocellular carcinoma in hepatic transplantation: value of lipiodol computed tomography. Transplant Proc. 1995; 27:2309-2310.

23. Barrett JC. Haploview: Visualization and analysis of SNP genotype data. Cold Spring Harb Protoc. 2009; 2009:pdb. ip71.

24. Schwartz S, Kent WJ, Smit A, Zhang Z, Baertsch R, Hardison RC, Haussler D, Miller W. Human-mouse alignments with BLASTZ. Genome Res. 2003; 13:103-107.
25. Siepel A, Bejerano G, Pedersen JS, Hinrichs AS, Hou M, Rosenbloom K, Clawson H, Spieth J, Hillier LW, Richards S, Weinstock GM, Wilson RK, Gibbs RA, et al. Evolutionarily conserved elements in vertebrate, insect, worm, and yeast genomes. Genome Res. 2005; 15:10341050 .

26. Rhead B, Karolchik D, Kuhn RM, Hinrichs AS, Zweig AS, Fujita PA, Diekhans M, Smith KE, Rosenbloom KR, Raney BJ, Pohl A, Pheasant M, Meyer LR, et al. The UCSC Genome Browser database: update 2010. Nucleic Acids Res. 2010; 38(Database issue):D613-619.

27. Ahn SM, Jang SJ, Shim JH, Kim D, Hong SM, Sung CO, Baek D, Haq F, Ansari AA, Lee SY, Chun SM, Choi S, Choi HJ, et al. Genomic portrait of resectable hepatocellular carcinomas: implications of RB1 and FGF19 aberrations for patient stratification. Hepatology. 2014; 60:1972-1982.

28. Fujimoto A, Totoki Y, Abe T, Boroevich KA, Hosoda F, Nguyen HH, Aoki M, Hosono N, Kubo M, Miya F, Arai Y, Takahashi H, Shirakihara T, et al. Whole-genome sequencing of liver cancers identifies etiological influences on mutation patterns and recurrent mutations in chromatin regulators. Nat Genet. 2012; 44:760-764.

29. Holm K, Melum E, Franke A, Karlsen TH. SNPexp - A web tool for calculating and visualizing correlation between HapMap genotypes and gene expression levels. BMC Bioinformatics. 2010; 11:600. 